\title{
Optimizing the microstructure of composite cathodes in garnet-based all solid-state batteries by continuum modeling and simulation
}

Moritz Clausnitzer, Simon Hein, Robert Mücke, Martin Finsterbusch, Timo Danner, Arnulf Latz

Due to their potentially higher energy densities and advantages in safety, all solid-state batteries (ASSBs) are being considered to replace conventional Li-ion batteries in a number of applications. Ceramic garnet-type electrolytes are promising candidates for the solid electrolyte in ASSBs because they provide high ionic conductivity and a wide electrochemical stability window.

To improve battery performance, the optimization of the composite cathode in ASSBs is crucial. The microstructure of the cathode as well as interface phenomena occurring between active material particles and electrolyte grains are performance-limiting factors which need to be improved.

In our contribution, we present microstructure resolved simulations in the 3D simulation framework BEST [1] demonstrating the effects of characteristic properties of the microstructure on the cell performance. By simulation studies using composite cathode microstructures with different particle size, active material fraction, and sinter density, we are able to identify advantageous properties which can serve as a target for the cell production.

In the experiments, a large polarization of garnet-based ASSBs is observed which is commonly attributed to secondary phase formation between the active material and electrolyte in the production process [2]. Therefore, a new electrochemical interface model is introduced to account for the hindered transport of $\mathrm{Li}$ and electrons due to secondary phases. This model is based on physical parameters of the interface and, thus, can be used to get insights into the interface properties which are still subject of intensive research [3].

[1] Latz, A.; Zausch, J. Thermodynamic consistent transport theory of Li-ion batteries. J. Power Sources 2011, 196, 3296-3302.

[2] Finsterbusch, M.; Danner, T.; Tsai, C.-L.; Uhlenbruck, S.; Latz, A.; Guillon, O. High Capacity GarnetBased All-Solid-State Lithium Batteries: Fabrication and 3D-Microstructure Resolved Modeling. ACS Applied Materials \& Interfaces 2018, 10, 22329-22339.

[3] Vardar, G.; Bowman, W. J.; Lu, Q.; Wang, J.; Chater, R. J.; Aguadero, A.; Seibert, R.; Terry, J.; Hunt, A.; Waluyo, I.; Fong, D. D.; Jarry, A.; Crumlin, E. J.; Hellstrom, S. L.; Chiang, Y.-M.; Yildiz, B. Structure, chemistry, and charge transfer resistance of the interface between Li7La3Zr2O12 electrolyte and LiCoO2 cathode. Chem. Mater. 2018, 30, 6259-6276 